\title{
无人驾驶中的场景实时语义分割方法
}

\author{
秦飞巍 1 ，沈希乐 ${ }^{1)}$ ，彭勇 ${ }^{1,2)}$ ，郡艳利 ${ }^{1)}$ ，袁文强 ${ }^{1)}$ ，计忠平 ${ }^{1)}$ ，白静 ${ }^{3)}$ \\ ${ }^{1)}$ (杭州电子科技大学计算机学院 杭州 310018) \\ ${ }^{2)}$ (苏州大学江苏省计算机信息处理技术重点实验室 苏州 215006) \\ 3) (北方民族大学计算机科学与工程学院 银川 750021) \\ (qinfeiwei@hdu.edu.cn)
}

\begin{abstract}
摘 要: 无人驾驶的一个重要组成部分是汽车行驶环境感知, 使人们对可在低功耗移动设备上实时运行的高精度语 义分割方法产生了强烈的需求. 然而, 在分析影响语义分割网络精度和速度的因素时可以发现, 空间信息和上下文 特征很难兼顾，而使用 2 路网络分别获取空间信息和上下文信息的方法，又会增加计算量及存储量. 因此，提出从残 差结构网络中划分出空间信息路径和上下文信息路径的想法，并基于此设计一个双路语义分割网络. 该网络还含有 用于融合 2 路多尺度特征的特征融合模块, 以及用于优化上下文语义路径输出结果的注意力精炼模块. 该网络基于 PyTorch 框架实现, 使用 NVIDIA 1080Ti 显卡进行实验，在道路场景数据集 Cityscapes 上, mIoU 达到 78.8\%, 运行速 度达到 27.5 帧/s.
\end{abstract}

关键词：无人驾驶；实时语义分割; 残差单元; 注意力机制

中图法分类号: TP391.41 DOI: 10.3724/SP.J.1089.2021.18631

\section{A Real-Time Semantic Segmentation Approach for Autonomous Driving Scenes}

\author{
Qin Feiwei $^{1)}$, Shen Xiyue ${ }^{1)}$, Peng Yong ${ }^{1,2)}$, Shao Yanli ${ }^{1)}$, Yuan Wenqiang ${ }^{1)}$, Ji Zhongping ${ }^{1)}$, and Bai Jing ${ }^{3)}$ \\ 1) (School of Computer Science and Technology, Hangzhou Dianzi University, Hangzhou 310018) \\ 2) (Provincial Key Laboratory for Computer Information Processing Technology, Soochow University, Suzhou 215006) \\ 3) (School of Computer Science and Engineering, North Minzu University, Yinchuan 750021)
}

\begin{abstract}
An important part of autonomous driving is the perception of the driving environment of the car, which has created a strong demand for high precision semantic segmentation algorithms that can be run in real time on low-power mobile devices. However, when analyzing the factors that affect the accuracy and speed of the semantic segmentation network, it can be found that in the structure of the previous semantic segmentation algorithm, spatial information and context features are difficult to take into account at the same time, and using two networks to obtain spatial information and context information separately will increase the amount of calculation and storage. Therefore, a new structure is proposed that divides the spatial path and context path from the network based on the residual structure, and a two-path real-time semantic segmentation network is designed based on this structure. The network contains a feature fusion module and an attention refinement module, which are used to realize the function of fusing the multi-scale features of two
\end{abstract}

收稿日期：2020-08-27; 修回日期：2020-12-07. 基金项目：国家自然科学基金(61972121，61971173，61702517，61802101, 61762003, U1909210); 苏州大学江苏省计算机信息处理技术重点实验室开放基金(KJS1841); 浙江省属高校基本科研业务费项目 (GK209907299001-008). 秦飞巍(1985-), 男, 博士, 副教授, 硕士生导师, CCF 会员, 主要研究方向为计算机辅助设计与图形学、机 器学习; 沈希乐 (1997一), 女, 学士, 主要研究方向为计算机视觉、机器学习; 彭勇(1985一), 男, 博士, 副教授, 硕士生导师, CCF 会 员, 主要研究方向为机器学习; 邵艳利(1989-), 女, 博士, 副研究员, 硕士生导师, CCF 会员, 主要研究方向为计算机辅助设计与图 形学、数据挖掘; 袁文强(1988-), 男, 博士, CCF 会员, 主要研究方向为计算机辅助设计与图形学; 计忠平(1980一), 男, 博士, 副教 授, 硕士生导师, CCF 会员, 主要研究方向为数字几何处理、机器学习; 白静(1982-), 女, 博士, 副教授, 硕士生导师, CCF 高级会员, 主要研究方向为计算机辅助设计与图形学、机器学习. 
paths and optimizing the output results of context path. The network is based on the PyTorch framework and uses NVIDIA 1080Ti graphics cards for experiments. On the road scene data set Cityscapes, mIoU reached $78.8 \%$, and the running speed reached $27.5 \mathrm{fps}$.

Key words: autonomous driving; real time semantic segmentation; residual unit; attention mechanism

随着人工智能与汽车交通的结合, “无人驾驶” 热潮被掀起. 无人驾驶 ${ }^{[1]}$ 也称为智能驾驶或自动驾 驶, 该项技术在车辆的自动行驶过程中通过计算 机策划行进路线, 控制车辆行动, 利用雷达、超声 波、全球定位系统(global positioning system, GPS)、 计算机视觉 (computer vision, CV)等技术识别多种信 号, 从而感知路况. 无人驾驶的诞生, 具有解放人类 身体、避免人为过失造成车祸、缓解交通拥堵与污 染等目的. 但是近年来, 无人驾驶汽车造成人员伤 亡、财物损失的交通事故也偶有报道. 安全成为无人 驾驶发展的第一要素, 因此, 如何做到精确又快速 地检测路况及道路标志等信息成为首要任务.

算法速度和精度提升是无人驾驶汽车对真实 场景的检测与识别的核心，只有高精度和处理快 速的语义分割网络才能让无人驾驶获取实时精确 的道路状况信息，从而提升无人驾驶的行驶安全 性与高效性. 然而, 现存网络大多无法兼顾速度和 精度，不是折损精度以换速度，便是在提升精度的 同时增大了计算量. 为了在实践中获得更好的行 驶表现，无人驾驶技术对语义分割算法的准确性、 实时性和稳健性提出了很高的要求, 当前应用于 无人驾驶的语义分割技术还存在着一定的难点.

由分辨率大的特征图空间信息较丰富, 分辨 率小而感受野大的特征图语义信息较丰富可见, 空间信息和上下文语义信息存在一定互斥性．考 虑现存网络对两者的顾此失彼，本文针对无人驾 驶情况中具体的分割任务，提出了双路语义分割 网络. 为了同时获取高质量的空间信息和上下文 信息，且尽可能地减少新增计算量及存储量，从而 提升速度，该网络模型从 ResNet-18 的不同阶段提 取输人图像的特征，利用空间信息分支和上下文 信息分支分别学习有效特征，设计了特征融合模 块(feature fusion module, FFM)和注意力精炼模块 (attention refinement module, ARM), 混合 2 种特征 并提升精度. 同时，在训练阶段使用一个主损失函 数和 2 个辅助损失函数相结合的方式, 在分别有效 地获取空间信息和上下文语义信息的结构下，最 终收获了较为突出的分割成效.

\section{1 相关工作}

\section{1 无人驾驶}

无人驾驶 ${ }^{[1]}$ 是一项涉及人工智能、雷达、GPS 和视觉计算等多项技术的前沿科技, 计算机在它 们的协作中能在没有人类操作的情况下自动完成 完整、安全和有效的驾驶. 它的社会价值在于能大 幅降低交通事故发生率、缓解目前拥堵的交通状况 和扩大驾驶主体范围.

在 1920 年前后，便已经可以看到无人驾驶汽 车相关研究的身影. 到 21 世纪时, 无人驾驶已有 数十年的研究历史. 现阶段, 无人驾驶作为人工智 能运用范畴内备受关注的热点, 相关技术的发展 如火如茶. 而无人驾驶也随着深度学习的进步趋 向实用化和商业化. 在中国, 无人驾驶受到强烈关 注，已经上升为国家战略. 谷歌、苹果和 Amazon 等科技领导者, Alphabet, Uber 和特斯拉等汽车制 造商, 以及 Oxbotica, Aurora.tech 和蔚来汽车等初 创企业都纷纷踏人自动驾驶汽车的研究与生产. 从国内外的研发状况的重视和投人来看, 无人驾 驶已累积大量经验，技术进步也呈高速增长趋势.

\section{2 图像语义分割}

语义分割是无人驾驶的核心科技之一，受到 了当下学术界和工业界等多个行业的重点关注.

\subsection{1 传统图像分割}

在深度学习发挥价值于图像分割以前，图像 语义分割的相关工作便层出不穷.

（1）基于阈值的分割．阈值分割法在传统图像 分割中是最基本且应用性最强的，其基本原理是 将像素点按照高于或低于设定的阈值区分为多个 区域，包括背景和多个目标区域. 基于阈值的方法 有直方图双峰法和 $p$-tile 法等, 选取阈值的方法有 直方图法、大津法 ${ }^{[2]}$ 和自适应阈值法等.

(2) 基于边缘的分割. 图像中像素变动梯度最 大的部分被定义为边缘 ${ }^{[3]}$ ，可以通过 Roberts， Canny 和 Laplacian 等边缘检测算子检测边缘，一 般是基于寻找图像一阶微商的极数或二阶微商的 零点的原理. 串行边缘检测和并行边缘检测是 2 类 
主要的边缘检测技术.

（3）基于区域的分割．其依据是像素区域相似 性，经典算法有区域增长和区域分裂兼并算法. 具 体来说，区域生长算法的原理是将像素按照性质 异同分别集合成若干区域 ${ }^{[3]}$ ，区域生长由小及大， 从部分像素点开始至整个区域; 而分离合并法则 相反, 它由大及小, 从完整图像中分离出子区域, 再按特征分别进行区域合并.

（4）基于图论的分割. 图论即以点代表物、以 边代表事物间的联系. 在基于图论的分割中，图像 像素点即是图中的点, 邻近像素间的特征相似度 即是图中的边, 通过对图中某些边的剪切将图分 离为互相独立的子图, 从而实现分割. 常用算法有 Graph $\mathrm{Cut}^{[4]}$ 和针对前者优化所得的 $\mathrm{Grab} \mathrm{Cut}{ }^{[5]}$ 等.

（5）基于能量泛函的分割. 其基本思想是将目 标边缘对应的连续曲线函数作为能量泛函的输人, 通过对该能量泛函求导获取的最小值则对应分割 的部分. 根据输人函数的性质, 可以将能量泛函分 割分为参数型和几何型 2 类.

\subsection{2 基于深度学习的语义分割}

基于深度学习的图像分割技术发展至今已经 出现了很多优质算法, 其中很大一部分都是在全 卷积网络(fully convolutional networks, $\mathrm{FCN})^{[6]}$ 这块 奠基石的基础上实现的. $\mathrm{FCN}$ 基于主流的卷积神经 网络(convolutional neural networks, CNN) ${ }^{[7]}$ 实现. 它将现有网络的目标从分类变为分割的操作是: 去掉分类网络中的全连接层, 转而代以一定数量 的卷积层和池化层.

2015 年, 剑桥大学提出的 SegNet 模型 ${ }^{[8]}$ 基于 先驱 FCN 实现了成功创新. 它是编码器-解码器结 构的创始者, 是现存语义分割算法中最流行的架 构. 编码器用于对输人提炼并获取其特征, 解码器 则连接在编码器后进行像素分类, 实现图像的分 割. 随后，更多语义分割算法相继问世，所实现的 分割精度也在一直攀升, 如 DeepLab 系列 ${ }^{[9]}$, RefineNet ${ }^{[10]}$ 和金字塔场景解析网络 (pyramid scene parsing networks, PSPNet $)^{[11]}$ 等.

深层神经网络在 $\mathrm{CV}$ 领域中是一个不可或缺 的角色，大部分神经网络虽然在理论和实验室实 验中收效极佳，但在实际运用中存在很大的局限 性. 以应用于无人驾驶的道路场景语义分割为例, 运用于现实情况的语义分割需要很强的实时性, 至少需要达到 10 帧/s, 这对计算量、参数量和存储 量都是很高的要求. 基于此, 实时语义分割逐渐演 化出来，如基于空洞卷积的 $\mathrm{ENet}^{[12]}$ 、谷歌提出的
MobileNet 系列、ICNet ${ }^{[13]}$ ，以及 Megvill 的 ShuffleNet 系列 ${ }^{[14]}$ 等.

当前, 语义分割中仍普遍存在几个亟待解决 的问题：(1) 最重要的便是分割精度的问题. 由于 追求处理速度, 大多数网络在无可奈何之下只能 舍弃对高精度的把握，导致实时语义分割网络的 分割精度普遍不如非实时语义分割网络，尤其是 在细小物体的分割上，轮廓的定位变得更加艰难; (2) 计算机资源的问题. 对高分辨率图像的高精度 分割对计算机资源要求极高, 运用轻量级网络的 效果普遍更为逊色, 这就要求足够的计算机资源, 如图形处理器 (graph processing unit, GPU), 而足 够的计算机资源又与无人驾驶这类移动应用场景 相矛盾，造成道路场景语义分割的实现更加困难; (3) 数据问题. 语义分割的数据集要求比目标检测 等更加繁杂, 不仅要标注出目标物体, 而且需要识 别出所有语义不同的类别, 是像素级的标注, 即使 是现在最流行丰富的数据集之一的 Cityscapes ${ }^{[15]}$, 也有许多未被标注的物体类别. 综上所述, 语义分 割中需要解决和改善的问题还很多，这将是影响 语义分割在生产生活中运用和普及程度的关键. 针对第 1 个算法速度与精度的问题, 诸多学者也在 努力推陈出新. 当前用于实现语义分割实施推理 速度的方法如下：

(1) 通过限定输人图像的大小进行加速. 该方 法的优点是简单有效地降低了计算复杂度, 缺点 是会损失不少空间细节。

(2) 通过删减通道数量进行加速. 这种方法常 用于 backbone 网络的初期, 劣势是会降低空间细 节的存在感.

(3) 通过抛弃模型的末尾部分进行加速. 以 $\mathrm{ENet}^{[12]}$ 为例, 该方法的缺点是最后阶段的丢弃导 致模型下采样程度不够深、感受野不够大, 因此判 别误差较大, 损失了模型性能.

以上方法优劣并存，在无人驾驶的语义分割 中, 想要兼得速度和精度还有很长的路要走.

\section{2 本文算法概述}

无人驾驶中进行语义分割的图像分辨率高, 对推理速度有很高的要求, 对于此类应用程序, 如 何维持高效推理速度和高精度并存是一个关键问 题. 很多方法采取损失分割性能换取快速处理速 度的方式, 因此本文为解决此痛点, 聚焦于实现一 个高精度的实时语义分割算法. 
为了实现以像素为单位给图像分配密集标签 的目的, CV 中采用语义分割任务进行图像分析. 该任务中，空间信息和上下文语义信息缺一不可， 空间信息对应像素的位置，对语义分割的物体边 界准确性很重要; 上下文语义信息对应像素的分 类, 对像素所属类别的判别很重要. 但是在 CNN 中, 这 2 个需求之间存在一定的矛盾. 在实时语义 分割的任务中, 为了提高推理速率, 各种方法层出 不穷, 有的缩小输人图像的尺寸, 有的使用轻量级 骨干模型，但是这 2 种方式一种减小了尺寸、一种 裁剪了通道，都丢失了部分空间信息. 总而言之， 空间分辨率和感受野不可兼得. 因为原始图像从 输人网络开始, 重复经历着卷积、池化的操作, 特 征图(feature map)的分辨率由大至小, 随着网络逐 渐进人较高的层次中，学习到的特征从空间信息 丰富逐渐进入语义信息更加丰富的阶段. 这样的 特征适用于图像分类等任务, 但语义分割不仅需 要分类, 还需高分辨率输出, 需要语义丰富的高层 级特征和空间信息丰富的低层级特征充分且有效 地融合，才能使网络性能更佳.

如第 1.2.2 节所述，当前用于实现语义分割实 时推理速度的方法主要有通过限定输人图像的大 小进行加速、通过删减通道数量进行加速, 以及通 过抛弃模型的末尾部分进行加速. 但这些方法会 丢失很多空间信息，从而导致精度大幅下降，即以 精度换速度, 无法在现实运用中取得理想效果. 针 对以上问题，为了补全缺失的空间信息，U 形结构 被提出并应用于语义分割，取得了不错的效果. U 形结构分为特征提取和上采样 2 部分, 在上采样的 每个层级引进相应的下采样阶段提炼的特征，通 过这种方式，U 形结构融合了多尺度特征，逐渐弥 补了空间信息. 但有得必有失, U 形结构相比普通 结构增加了不少计算量, 导致网络处理速度减缓, 无法达到实时的要求; 另外, 以这种操作补回的空 间细节微不足道, 大部分细节信息无法轻易通过 不同层级特征的融合找回. 除了 U 形结构, 也有方 法采用多分支框架，通过组合空间细节信息和上 下文语义信息解决问题. 然而，与 U 形结构相似, 新增的分支给整个网络带来了多余的计算量, 导 致速度变慢, 更重要的是模型的学习能力被分支 之间的相互独立性限制住了. 除此之外, 还有利用 空洞卷积、空间池化金字塔等方法的网络，但是此 类方法会限制速度, 且需要出色的计算能力, 轻量 级骨干网络无法胜任.

基于上述各类模型的优缺点，本文设计了双
路语义分割网络，以一种新方法同时保证空间细 节和上下文语境. 在双路语义分割网络中, 以残差 网络为基础，分出空间信息分支和上下文信息分 支 2 部分, 输出不同分辨率的特征图. 上下文信息 分支用于获得足够小的感受野, 实现特征提取; 空 间信息分支用于弥补分辨率细节，解决空间信息 损失的问题. 本文也提出了 FFM 和 ARM, 用于来 自不同分支的特征融合和输出的优化.

\section{3 双路语义分割网络}

在以 ResNet-1 $8^{[16]}$ 为骨干网络的基础上，本文 提出的双路语义分割网络分流出 2 个分支, 分别用 于获取空间信息和上下文信息. 从一定角度看，该 网络借鉴了 PSPNet ${ }^{[1]}$ 的多尺度特征融合概念. 与 其他网络相比, 本文的双路语义分割网络在前人基 础上提升了性能, 实现了高精度的实时分割.

\section{1 整体结构}

图 1 所示为本文提出的双路语义分割网络架 构, 其中的骨干网络是一个不完整的 ResNet-18(平 均池化层前面的部分). 整个模型以残差网络为基 础, 利用残差网络 ResNet-18 不同阶段的输出收集 不同尺度的信息, 用于实现高精度的语义分割. 在 ResNet-18 的每个阶段中学习到的特征都不同, 前 期阶段的特征图分辨率大，学习到的特征包含的 空间信息比较丰富; 随着网络趋向深层, 其感受野 变大, 学习到的特征层次变高, 语义信息变得丰 富. 在分类任务中, 图像语义信息对提高准确性至 关紧要. 但在语义分割中, 仅实现精确分类是不够 的, 还需要足够的空间信息才能得到高精度的分 割结果. 基于此, 本文网络采用不同的分支进行不 同信息的获取. 图 2 所示为网络的组成细节图, 可

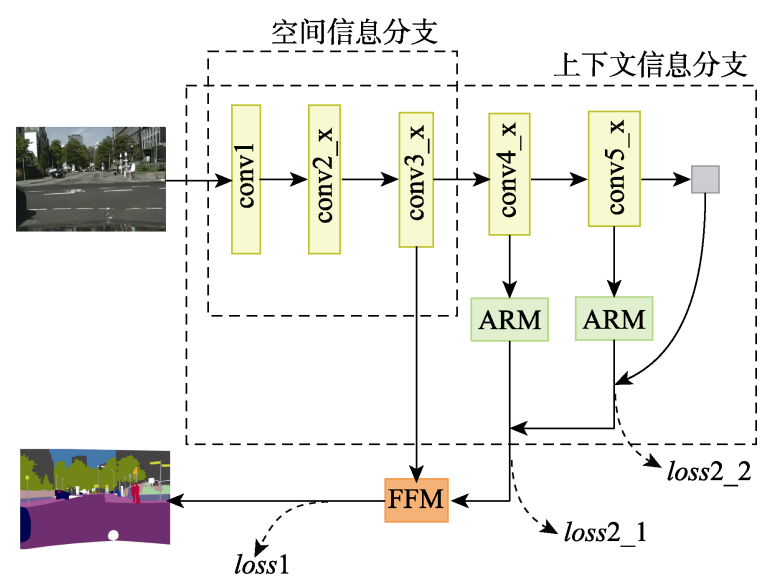

图 1 双路语义分割网络结构 
以看出, 它是经典的编码器-解码器结构.

由图 1 和图 2 即可清楚地观察到, 该模型架构 含有一个骨干网络，从中分出了 2 个信息流分支, 分别是空间信息分支和上下文信息分支. 其中，用
于空间信息分支的输出特征图为网络输人大小的 $1 / 8$, 为网络保留充足的空间信息; 用于上下文信 息分支的输出特征图分别为网络输人的 $1 / 16$ 和 1/32, 用于获取高级上下文信息.

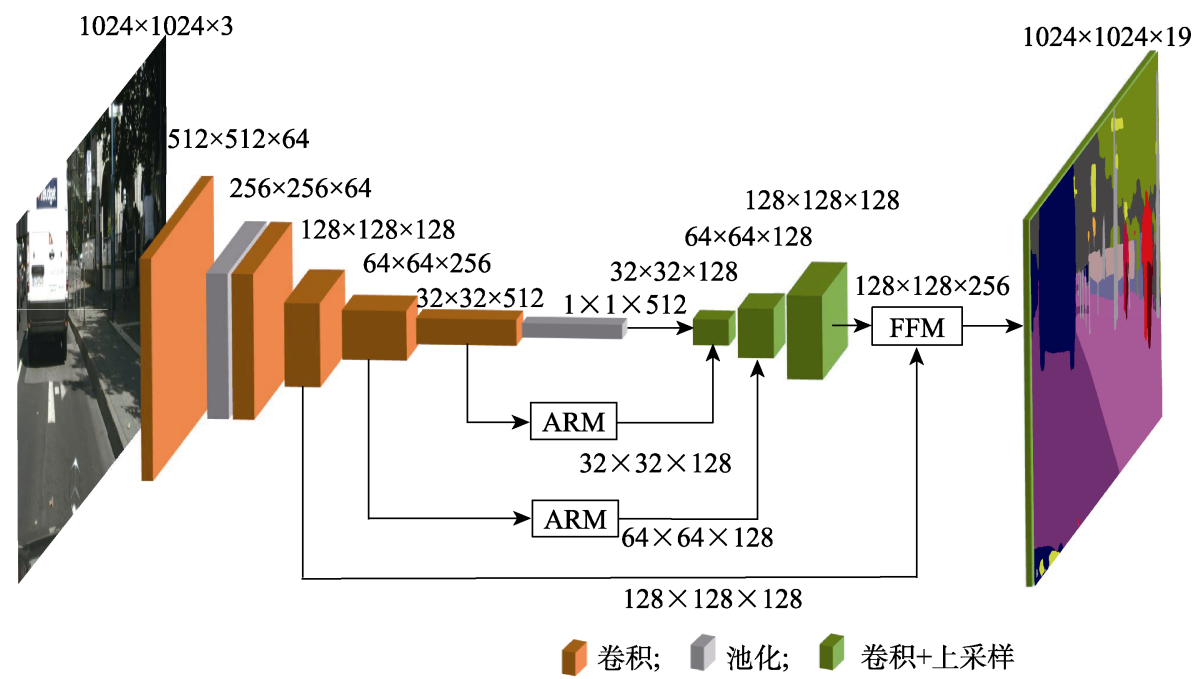

图 2 双路语义分割网络组成细节

本文网络特地设计了 FFM，用于融合各个分 支的输出; 然后在对 FFM 的输出直接上采样至原 图大小之后, 输出结果.

\section{2 空间信息分支}

在语义分割任务中，要想获得精确分割的效 果, 需要将高层次的丰富语义信息和低层次的空 间信息结合起来，但对实时语义分割情形中的这 两者来说, 很容易顾此失彼. 鉴于此, 本文提出额 外加人网络的空间信息分支, 用于弥补语义分割 中丢失的空间信息. 图 1 中, 空间信息分支包含了 残差网络 ResNet-18 的前 3 层, 也就是利用其第 3 阶段 conv3_ $x$ 的输出特征, 说明此输出的空间尺寸 为原图的 $1 / 8$. 此阶段的特征分辨率较高, 并且已 经学习到了足够的空间信息. 后续该输出与上下 文信息分支的特征进行融合.

\section{3 上下文信息分支}

为获取足够的语义信息，一些方法提出了金 字塔池化 (spatial pyramid pooling, SPP) 模块 ${ }^{[17]}$ 、金 字塔形空洞池化 ${ }^{[9]}$ (atrous spatial pyramid pooling, ASPP)或较大卷积核增大感受野, 但实时语义分割 网络对计算速度要求较高, 而这些操作既耗时又 耗内存, 很难满足条件. 为了兼顾大感受野和高效 率, 本文设计了上下文信息分支, 在利用轻量级网 络快速下采样后, 添加全局平均池化 (global average pooling, GAP)以最大化感受野, 获取了全局信 息. 基于各种考虑，上下文信息分支以残差网络
ResNet-18 作为骨干网络, 用于获取高级上下文特 征, 进而改善语义分割网络的分类能力. 图 1 中, 上下文信息分支即在空间信息分支的基础上继续 下采样，与空间信息分支共存于一路网络. 该分支 中, 主角是 ResNet-18 最后 2 个阶段的输出, 空间 尺寸分别为原图的 $1 / 16$ 和 $1 / 32$. 在骨干网络的最 后, 为了最大化网络的感受野, 还加人了全局平均 池化, 将特征图空间尺寸变成了 $1 \times 1$ 像素. 将此特 征图上采样后与经过 ARM 后的骨干网络的最后输 出在通道上简单叠加后, 再与经过 ARM 的骨干网 络倒数第 2 阶段的输出相加, 然后作为上下文信息 分支的输出与空间信息分支的输出进行融合.

\subsection{ARM}

ARM 用于上下文信息分支, 以提炼最后 2 个 阶段的输出. ARM 仿照 $\mathrm{SENet}^{[18]}$ 使用注意力机制 的方式, 在开头使用 GAP 获得最大感受野, 从而 整合全局上下文语义信息; 在后续步骤中，通过注 意力掩码训练网络侧重性地学习, 使特征带有不 同的权重. 具体地, ARM 针对特征图的每个通道 计算其权重, 然后对每个原来的输出通道用对应 的权重进行加权, 得到新的加权后的特征, 起到重 新调节整合特征的作用. 该设计采用注意力机制, 达到使用极少计算量便对上下文信息分支中 2 个 阶段(即 ResNet-18 下采样的第 4 阶段和第 5 阶段) 的输出进行提炼优化的效果, 简单快速地获取全 局上下文语义信息。 
如图 3 所示，在 ARM 中通过一系列操作得到 特征图每个位置对所有位置的响应权重矩阵, 然 后使用 sigmoid 将权值映射至 $0 \sim 1$, 再将权值与特 征相乘，得到加权后的响应特征.

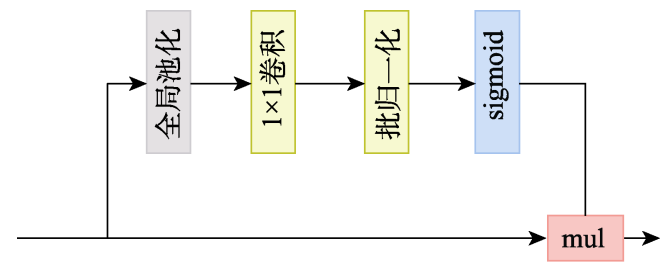

图 $3 \mathrm{ARM}$ 结构

\subsection{FFM}

空间信息分支捕获的特征中空间细节更充足， 上下文信息分支捕获的特征中包含着丰富的语境 信息，其输出的特征一个较浅层、一个较深层，并 不是同一个层级的，因此无法直接对其进行合并， 而是需要一个专门融合这些不同尺度特征的独特 模块，即本文设计的 FFM.

如图 4 所示，在被 2 个分支给定了级别参差的 特征的情况下, FFM 学习注意力掩码用来选择和 结合特征. 首先对不同的输人特征直接使用 concatenate 串联后进行常规的卷积-批归一化-ReLU 操作; 接下来进行特征优化, 与 ARM 类似, 通过 全局平均池化获得串联后特征的特征向量，通过 卷积和激活函数计算不同特征的权重; 再通过权 重与特征相乘重新加权特征; 最后与原特征相加.

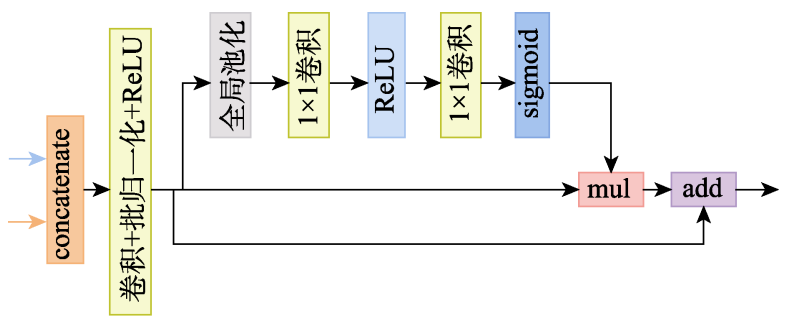

图 4 FFM 结构

\section{6 损失函数}

双路语义分割网络的 2 个分支各自学习不同 层次的特征，并且其中的上下文信息分支对网络 整体的性能十分重要, 充足的上下文语义信息才 能减少同类别物体被分割为多个部分或不同类别 物体被分割为同一部分的情况发生. 为了更好地 监督网络训练，本文为网络的上下文信息分支添 加了辅助损失函数. 除监督整个网络输出的主损 失函数外，在上下文信息分支中添加了 2 个辅助损 失函数监督其输出. 3 个损失函数的具体位置如图
1 中的虚线路径所示, 即主损失函数 loss1 、辅助损 失函数 loss2_1 和 loss2 2 2. 其中各损失函数的操作 流程是：分支的输出经过卷积-批归一化-ReLU 平 衡了特征尺度之后, 通过单位卷积将特征图的通 道数改为类别数目, 即 19 , 最后用双线性插值法 对特征图进行上采样至输人大小后计算其损失.

3 个损失函数以简单地相加结合在一起，即

$$
\text { loss }=\operatorname{loss} 1+\operatorname{loss} 2 \_1+\operatorname{loss} 2 \_2
$$

其算法统一, 都是结合了难例挖掘 (online hard example mining, OHEM) 策略 ${ }^{[19]}$ 的交叉熵损失函数, 如

$$
L=\frac{1}{N} \sum_{i} L_{i}=\frac{1}{N} \sum_{i}-\sum_{c=1}^{M} y_{i c} \ln \left(p_{i c}\right)
$$

其中, $M$ 指类别数量; $y_{i c}$ 值为 0 或 1 , 若类别与样 本 $i$ 相同, 则为 1 , 否则为 $0 ; p_{i c}$ 为样本 $i$ 属于类别 $c$ 的预测概率.

\section{4 实验与结果分析}

本文实验基于深度学习框架 PyTorch 进行实 现，还使用由 NVIDIA 推出的通用并行计算架构 (compute unified device architecture, CUDA)加速 GPU 运算; 使用 Numpy 等 Python 包进行数据处理, 使用 Matplotlib 库实现分割结果可视化等操作.

\section{1 训练设置}

\section{1 .1 数据集预处理}

本文实验使用 Cityscapes 数据集作为用于训 练和测试的数据集, 其中, 训练集为 2975 张图片, 验证集为 500 张图片. 在训练中, 为了扩充数据集, 对训练集实施预处理, 采取对图像进行标准化、随 机缩放、随机裁剪、水平翻转, 以及对图像的亮度、 对比度和饱和度进行调整等方式实现数据增强. 实验中涉及的参数设置说明如下: Cityscapes 数据 集图像的原始尺寸为 $2048 \times 1024$ 像素，经随机裁 剪后尺寸为 $1024 \times 1024$ 像素; 随机缩放的尺度列 表为 $(0.75,1.00,1.25,1.50,1.75,2.00)$; 在颜色的 数据增强中, 亮度、对比度和饱和度 3 个参数均为 0.5 , 即图像对应的增强因子在 $0.5 \sim 1.5$ 随机选取; 另外，在测试中采取对验证集进行不同尺度的缩 放后再预测的方式，对分割物体的大小差别拥有 很好的容错性，提升了精度.

\section{1 .2 组合多种训练优化策略}

(1) Poly 策略 ${ }^{[9]}$. 学习率是一个对模型权重更 新具备极大影响力的超参数. 初始学习率设置合 理才能使模型最优化，过小会导致收敛慢，过大则 
会导致不稳定或收敛失败. 学习率需要随着网络 训练程度变化, 其变更策略很重要, 在深度学习中 存在多种策略, 如 Fixed 策略、Poly 策略和 sigmoid 策略. 本文实验的 SGD 优化策略中添加了 Poly 学 习率衰减策略，当前学习率为

$$
r_{\text {cur_l }}=r_{\text {init_ } 1} \times\left(1-\frac{n_{\text {it }}}{i_{\text {max } \_ \text {it }}}\right)^{p}
$$

其中, $r_{\text {init } 1}$ 为初始学习率, 设置为 $0.01 ; n_{\mathrm{it}}$ 为当前 迭代次数; $i_{\text {max it }}$ 为最大迭代次数, 设置为 $80000 ; p$ 用于控制曲线的形状，设置为 0.9 .

(2) Warmup 策略 ${ }^{[16]}$. 深度学习中, 模型的初始 权重是在训练启动阶段随机生成的, 选择 Warmup 策 略可以使模型在训练初期使用较小的学习率进行训 练, 经过设定的一定数量的迭代次数, 模型趋向稳 定后，再改为预先设定的学习率，达到预热学习率 的效果，可以防止模型震荡，加速网络收玫速度， 提升效果。

实验中使用 Warmup 策略中的 Gradual Warmup, 即在学习率预热阶段中学习率随着迭代次数增加 也逐步提高, 直到预热阶段结束时学习率达到预 设定值, 再进行后续训练, 这样能够避免学习率突 然增大而导致训练误差激增的情况.

参数设置如下：使用 Warmup 策略的迭代次数 为 1000 , 最初学习率及预热后学习率分别是 $10^{-5}$ 和 $10^{-2}$. 即模型在训练前期的 1000 次迭代中，学 习率将从 $10^{-5}$ 逐步增长至 $10^{-2}$, 再使用 Poly 策略 进行后续的训练. 在加人了 Warmup 策略后的训练 中, Poly 策略公式修改为

$$
r_{\text {cur_l }}=r_{\text {init_1 }} \times\left(1-\frac{n_{\text {it }}-s_{\text {warmup }}}{n_{\text {max_it }}-s_{\text {warmup }}}\right)^{p}
$$

(3) $\mathrm{OHEM}^{[19]}$. 其中, 网络会自动选择有多样 性和高损失的困难样本作为训练样本. 实验证明, OHEM 算法能高效率地改善网络参数. 为了进行 难例针对性学习, 本文网络使用交叉熵损失函数 和 OHEM 结合的方式进行训练. 通过 OHEM 策略 选取一定数量即 $n$ 个困难样本：按 loss 值降序排序 后, 若大于阈值的样本数多于 $n$, 则选择所有大于 阈值的样本; 否则, 按序选择前 $n$ 个样本.

将 OHEM 的阈值设为 0.7 , 则

$$
n=\frac{\text { batchsize } \times \text { cropsize _ } 0 \times \text { cropsize _ } 1}{16}
$$

其中，cropsize_0 和 cropsize _ 1 指输人图像的长和 宽; batchsize 指单次训练样本数.

\subsection{3 网络参数初始化}

实验网络中使用的骨干网络是不囊括最后全 连接层的 ResNet-18, 从 PyTorch 官方下载该网络 的预训练模型，即使用其在 ImageNet 上预训练后 的权重. 对于网络的其他部分, 各参数初始值设置 如表 1 所示.

表 1 网络参数初始值设置

\begin{tabular}{ll}
\hline \multicolumn{1}{c}{ 参数名称 } & \multicolumn{1}{c}{ 数值 } \\
\hline 输人分辨率 & $1024 \times 1024$ \\
batchsize & 8 \\
迭代次数 & $8 \times 10^{4}$ \\
学习率(预热前) & $10^{-5}$ \\
学习率(预热后) & $10^{-2}$ \\
\hline
\end{tabular}

\section{2 结果评估}

\subsection{1 有效性验证}

本文在双路语义分割网络中提出了多个模块 和方法, 需要对各部分进行有效性验证, 从而清楚 地观察到各部分对模型整体性能提升的作用.

各次实验在 Cityscapes 训练集上进行模型训 练后, 于验证集上进行各指标测试. 各参数设置与 整体精度测试实验保持一致. 结果数据如表 2 所

\begin{tabular}{|c|c|c|c|c|c|c|}
\hline FFM & 串联 & ARM & loss 1 & loss2_1 & loss2_2 & $\mathrm{mIoU} / \%$ \\
\hline V & & V & V & V & V & 78.80 \\
\hline V & & V & V & V & & 77.68 \\
\hline V & & V & V & & V & 77.81 \\
\hline V & & V & V & & & 77.65 \\
\hline V & & & V & V & V & 78.02 \\
\hline & V & V & $V$ & $V$ & V & 76.61 \\
\hline
\end{tabular}
示，部分可视化对比如图 5 所示.

表 2 有效性验证

注. 粗体部分即本次实验的极优值.

具体分析如下: 包含各部分的完整网络的数据 位于表 2 第 1 行, 可视化结果如图 5a 所示, 作为参 照基准用于对比; 表 2 的第 2 4 行是对辅助损失函 数进行有效性验证的 3 个实验, 分别是只包含主损 失函数、包含主损失函数和其中 1 个辅助损失函数 的情况，可以看出, 3 个损失函数结合的实验 $\mathrm{mIoU}$ 结果是最好的, 任意一个辅助函数的存在都是很 有必要性的, 它们为模型性能提升了至少 $1 \%$ 的 mIoU. 相应地，只包含一个主损失函数的模型的 分割结果如图 $5 \mathrm{~b}$ 所示, 可以看出, 分割结果在细 节上更为粗楉, 遗漏与错判的情况更多, 如标志 


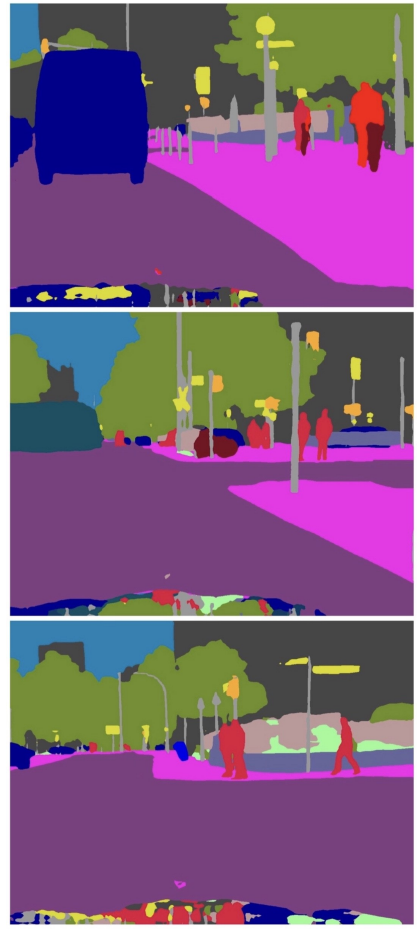

a. 完整模型
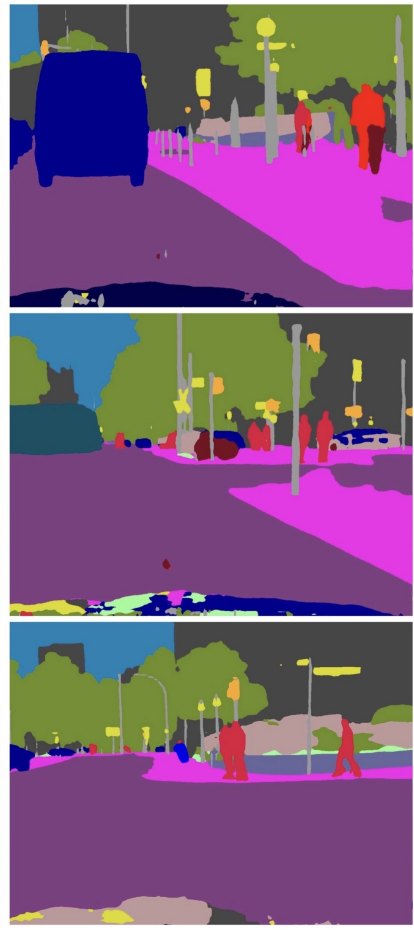

b. 主辅助损失函数

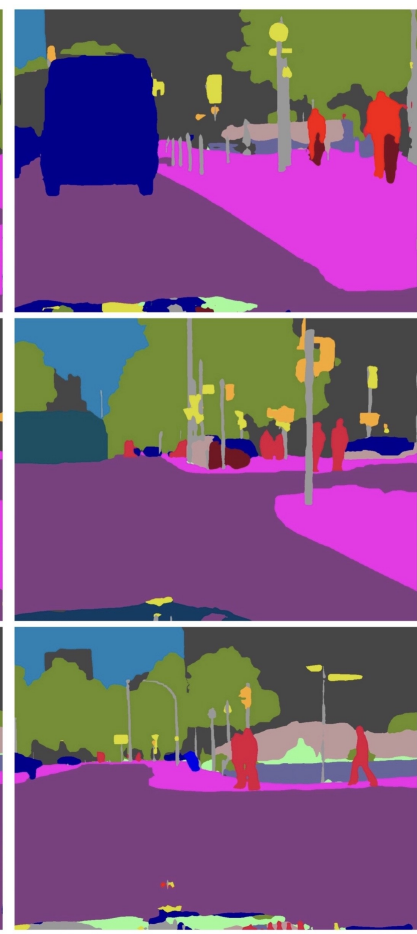

c. 不含 ARM

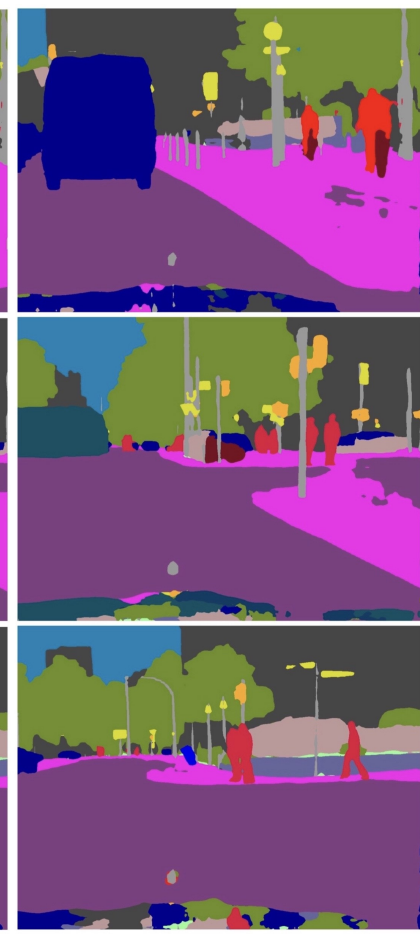

d. 不含 FFM

图 5 有效性验证

牌; 表 2 的第 5 行是针对 ARM 的有效性验证, 相 应的分割结果可视化图如图 5c 所示, 在不使用 ARM 的情况下, 为了使实验正常进行, 对上下文 信息分支的 2 个阶段的输出分别直接采取 $1 \times 1$ 卷积 改变通道数的简单方式，由性能结果对比可知，它 将网络的 $\mathrm{mIoU}$ 提升了约 $0.7 \%$, 说明 ARM 是有效 的，这从可视化图中马路边沿等多处细节都可以 看出; 表 2 的第 6 行及图 $5 \mathrm{~d}$ 是针对 FFM 的有效性 验证，本次实验中，对于空间信息分支和上下文信 息分支输出特征的融合采用直接串联融合代替 FFM 的方式, 由结果对比可知, FFM 为网络带来了 $2 \%$ 的性能提升, 是所有有效性实验中对模型改善 性能最好的，由分割图也可以看出，如无 FFM 的 模型在步行道的整体分割中误判最多. 总而言之, 以上实验成功地验证了各模块的必要性.

\subsection{2 性能测试与对比}

本节选取部分经典网络作为基准评判模型进 行性能的对比及分析, 包含 DeepLab 系列等非实 时语义分割网络. 表 3 中, 最后一行的“本文”表示 本文提出的双路语义分割网络.

（1）分割精度. 在测试准确性实验中，输人分 辨率为 $1024 \times 1024$ 像素. 准确性的测算与对比结 果如表 3 所示. 可以看出, 本文网络在准确性上明 显优于其他网络. 用于对比的网络中还包含基础
表 3 准确性测试与对比

\begin{tabular}{llc}
\hline \multicolumn{1}{c}{ 模型 } & Base Model & $\mathrm{mIoU} / \%$ \\
\hline Dilation10 $^{[20]}$ & VGG-16 & 68.7 \\
LRR $^{[21]}$ & VGG-16 & 70.0 \\
DFANet $^{[22]}$ & Xception & 71.3 \\
DeepLab-v2+CRF $^{[9]}$ & ResNet-101 & 71.4 \\
RefineNet $^{[10]}$ & ResNet-101 & 73.6 \\
DUC $^{[23]}$ & ResNet-152 & 76.7 \\
BiSeNet $^{[24]}$ & ResNet-18 & 78.6 \\
本文 & ResNet-18 & $\mathbf{7 8 . 8}$ \\
\hline
\end{tabular}

注. 粗体表示当前条件下最优值.

网络更加强大的模型, 如以 ResNet-101 为基础网 络的 DeepLab-v2+CRF 和以 ResNet-152 为基础网 络的 DUC, 它们在本文实验条件下达到的 mIoU 分 别为 $71.4 \%$ 和 $76.7 \%$, 而本文网络在以 ResNet- 18 为基础网络的情况下仍然更胜一筹, 提升了至少 $2 \%$ 的 mIoU, 取得了 $78.8 \%$ 的高精度.

(2) 分割速度. 在测试速度实验中, 速度越快, 表明网络越顺畅. 实验的输人分辨率为 $1024 \times 2048$ 像素. 速度的测试与对比结果如表 4 所示, 对应的 散点图如图 6 所示, 其中, 用于在 NVIDIA 1080Ti 上进行测试的 BiSeNet(Res18)为个人实现版.

从表 4 可以看出, 本文网络充分满足实时语义 分割的要求, 并且在输人分辨率为 $1024 \times 2048$ 像素 
表 4 速度测试与对比

\begin{tabular}{|c|c|c|c|c|}
\hline \multirow{2}{*}{ 模型 } & \multirow{2}{*}{$\begin{array}{c}\text { 输人 } \\
\text { 分辨率/像素 }\end{array}$} & \multicolumn{3}{|c|}{ 速度/帧· $\left.\cdot \mathrm{s}^{-1}\right)$} \\
\hline & & $\begin{array}{c}\text { NVIDIA } \\
\text { Titan X } \\
\end{array}$ & $\begin{array}{l}\text { NVIDIA } \\
\text { Titan XP } \\
\end{array}$ & $\begin{array}{c}\text { NVIDIA } \\
\text { 1080Ti }\end{array}$ \\
\hline SegNet $^{[8]}$ & $640 \times 360$ & 14.6 & & \\
\hline $\operatorname{ENet}^{[12]}$ & $1080 \times 1920$ & 21.6 & & \\
\hline $\mathrm{SQ}^{[25]}$ & $1024 \times 2048$ & 16.7 & & \\
\hline ICNet $^{[13]}$ & $1024 \times 2048$ & & 30.3 & \\
\hline Two-columnNet ${ }^{[26]}$ & $1024 \times 2048$ & & 14.7 & \\
\hline $\mathrm{BiSeNet}(\operatorname{Res} 18)^{[24]}$ & $1080 \times 1920$ & 23.0 & 34.4 & \\
\hline BiSeNet(Res18) $)^{[24]}$ & $1024 \times 2048$ & & 65.5 & 9.1 \\
\hline 本文 & $1024 \times 2048$ & & & 27.5 \\
\hline
\end{tabular}

注. 粗体表示每个模型在不同条件下的最大速度.

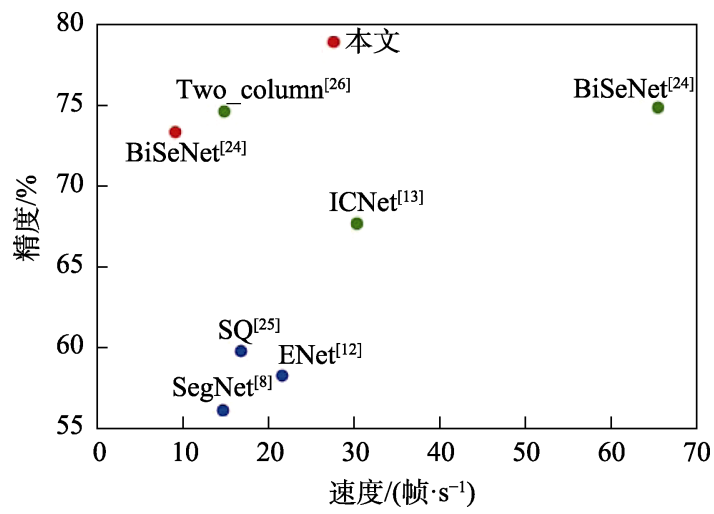

图 6 各模型速度与精度对比

和 NVIDIA 1080Ti 的显卡条件下，达到了 27.5 帧/s, 比其他多个模型的速度更快，甚至在其他模型处 于更小输人分辨率及更优性质的显卡条件的情况 下，如在 NVIDIA Titan X 上运行、输人分辨率为 $1080 \times 1920$ 像素的 BiSeNet(Res18). 在图 6 中, 蓝色 圆点表示该数据的实验设备环境是 NVIDIA Titan X, 绿色圆点表示 NVIDIA Titan XP, 红色圆点表示 NVIDIA 1080Ti. 可以看出, 双路语义分割模型的 精度与速度兼得性更加优秀. 总之, 双路语义分割 网络的分割速度已经获得了突出的成绩.

\subsection{3 可视化结果}

本文提出的双路语义分割网络在 Cityscapes 数据集上的部分分割结果可视化及与 BiSeNet 模 型可视化对比如图 7 所示.

首先，观察原始图像并对比地面标注图与实 际分割图(即前 3 列的图像)可以看出，本文提出的 双路语义分割模型总体表现优秀，优于当前大部 分实时语义分割模型. 详细分析如下：双路语义分 割网络对不同类别的标注效果不同. 其中, 对车 辆、树木与树丛、天空、行人、马路边缘, 以及房 屋建筑等空间占比大类别的分割效果较为优异,
基本都能准确识别其所属类别, 并且分割边缘也 较为光滑、准确; 但是对于交通标志牌、路杆、树 干、围栏等相对细小的物体分割效果不够理想，经 常出现遗漏、识别错误、边缘粗粘等问题，如最后 1 组图片中右侧的路灯集中部分, 大多数路杆都未 被识别出来. 虽说大型物体的分割较为准确, 但从 细节上看也存在部分较大的瑕疪, 如第 3 组图中行 人密集部分, 很多行人的肢体都未被精确分割. 此 外, 从结果可视化图中对白色类别部分的分割可 以看出, 由于缺少标签训练, 网络对未标注物体的 分割非常混乱, 如每幅图像下方车辆的车盖及车 标部分, 这也是语义分割中不可避免的问题.

其次，用于本次可视化对照的 BiSeNet 是在相 同实验条件下实现的，且同样基于基础网络 ResNet-18. 观察比较 2 个模型的实际分割图(即最 后 2 列图像)可知, 双路语义分割网络的分割效果 精确于 BiSeNet, 具体区别如下: (1) 在大型物体的 分割上, 本文网络分割更准确精细、边沿光滑, 以 第 1 组图道路中间的货车为例, BiSeNet 的分割图 中深蓝色(即车辆)中夹杂着一小部分黄色 (即标志 牌)的错误识别; 第 4 组图中对马路边缘的分割, 明显可见双路语义分割网络的划分边沿更加光滑. (2) 在小型物体的分割上, 本文网络的识别与分类 更加正确. 如在第 3 组图中, 本文网络成功地识别 了部分电车上方的电缆, 而 BiSeNet 发生了识别遗 漏; 第 4 组图中, 本文网络对右上角部分的 2 个路 杆进行了正确分类，而 BiSeNet 发生了分类错误， 将其顶部的突出部分归类为了标志牌. 简单对比 本文网络与 BiSeNet 的结构可以看出, 虽然同样是 分别获取图像的空间信息和上下文信息，但 BiSeNet 中的 2 个分支是独立的，新增的分支给整 个网络带来了多余的计算量, 导致速度变慢, 且模 型的学习能力被分支之间的相互独立性限制.

总体而言，本文网络在无人驾驶场景中取得 了较好的语义分割效果, 优于多个现存网络.

\subsection{4 视频语义分割}

在实际情况中, 无人驾驶以视频流的形式连 续捕捉道路情况是更佳的选择. 为了更好地运用 于实际且检验本文网络的性能, 本次实验对该网 络实现了针对视频的语义分割. 用于实验的视频 为日常交通道路视频，获取于 Youtube 网站.

具体步骤如下：首先针对原视频进行逐关键 帧提取, 输人预训练的双路语义分割网络进行分 割, 再将可视化后的分割图作为关键帧写人视频 


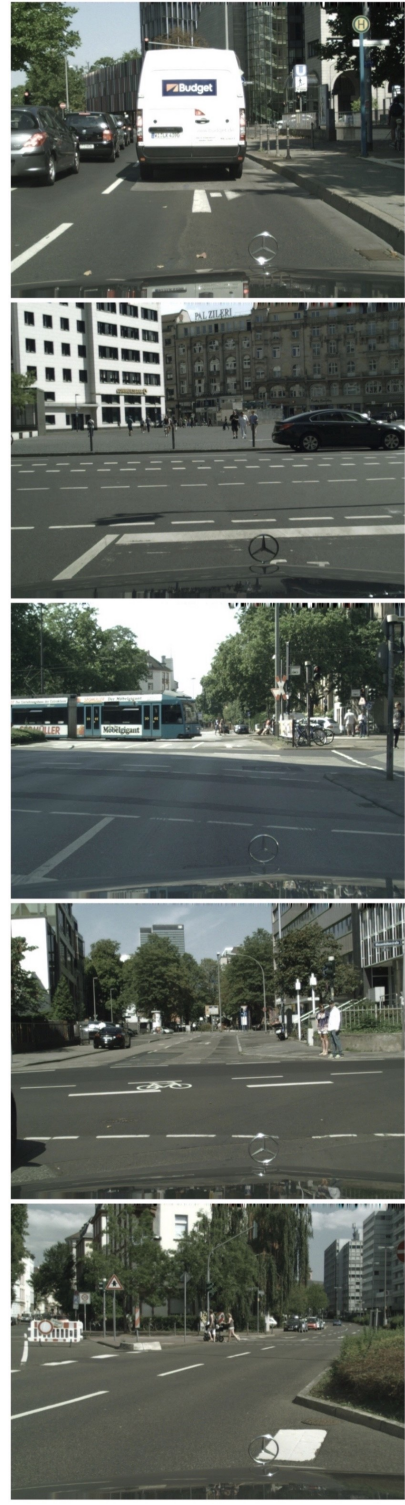

a. 原图像
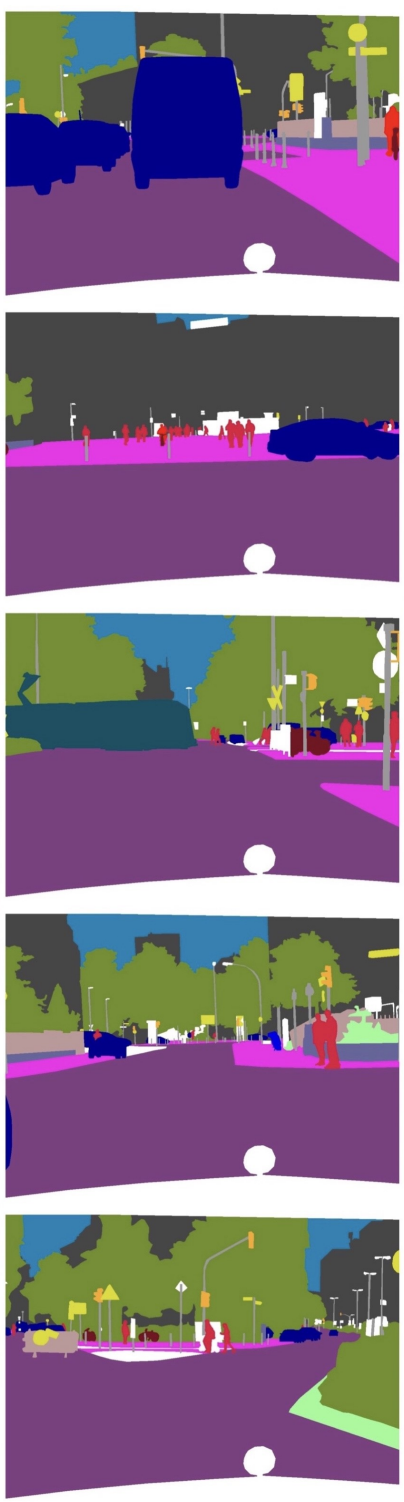

b. Ground Truth

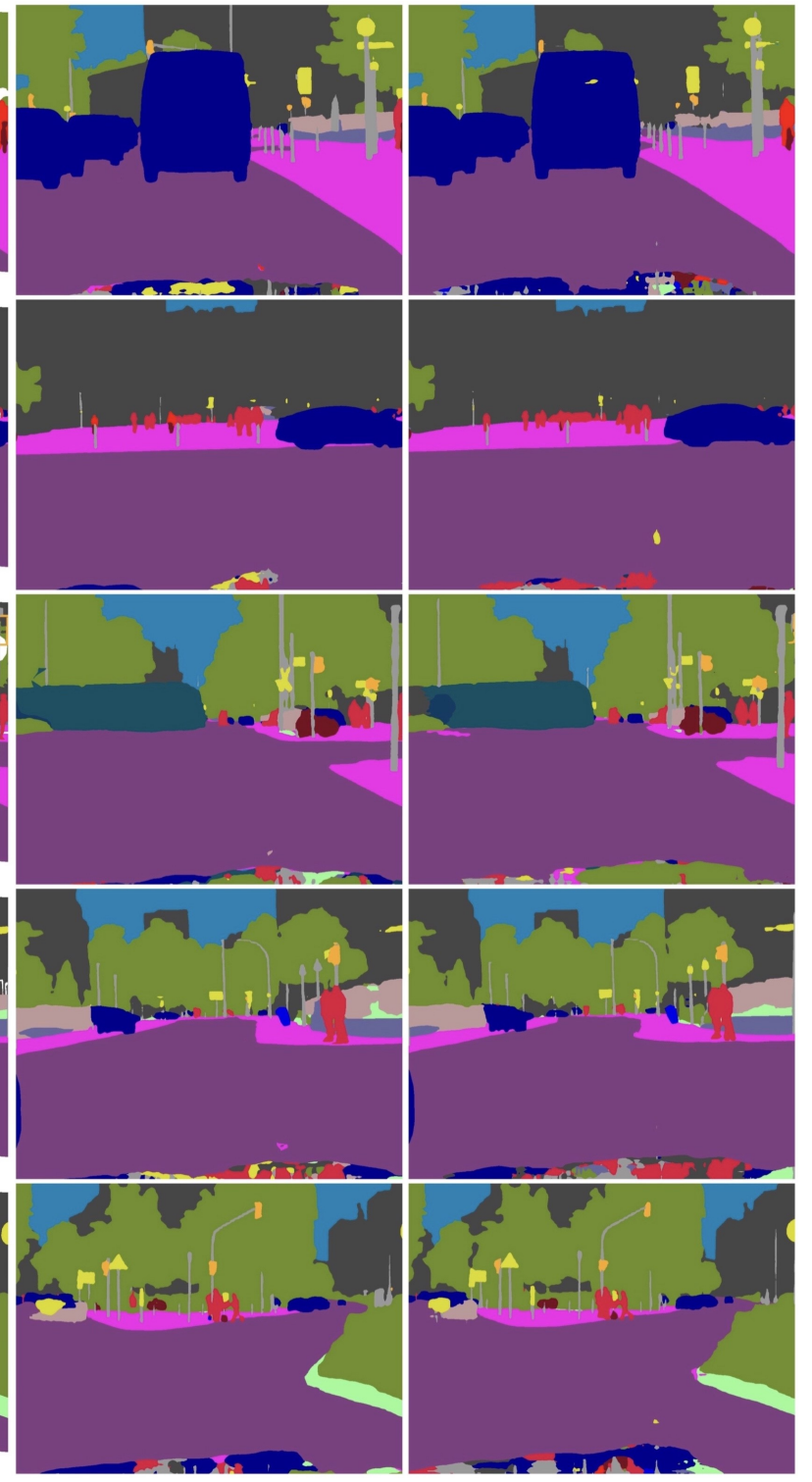

c. 双路语义分割网络

d. $\mathrm{BiSeNet}{ }^{[24]}$

图 7 部分分割结果

流，便获得针对原视频进行语义分割后的视频. 其 中, 部分原视频的关键帧及其相应的分割关键帧 展示如图 8 所示.

观察原始关键帧并对比分割图可以发现，双 路语义分割网络在视频语义分割中表现良好. 大 体上看, 大部分的大型物体基本被正确分割并分 类, 且轮廓较完整准确, 如汽车、行人、道路, 但 是也存在部分较为严重的错误, 如图 8 中前 2 组图 左上部分的天空被误判成了建筑物; 相比之下, 细 小物体的分割误差更大一些. 相对于 Cityscapes 的 验证集静态图, 语义分割网络对视频的关键帧分 割精度更低, 对比本文网络及 BiSeNet 的视频分割 可视化结果可以看出, 本文网络的分割效果仍优
于 BiSeNet. 主要原因如下: (1) 双路语义分割网络 使用 Cityscapes 训练集进行训练, 验证集中的图片 与之风格相似, 分割更加准确, 而视频随机选自网 络, 清晰度、场景风格和物体类别等指标都无法确 定, 且与 Cityscapes 数据集差距较大, 需要考虑模 型的泛化能力; (2) 使用 Cityscapes 验证集进行模 型测试时, 采取缩放裁剪等方式处理图像来提升 精度, 在视频语义分割中却是直接对提取出的关 键帧进行分割, 因此在精度上也打了折扣. 基于以 上原因, 视频分割的效果或多或少更加不理想, 但 总体上已经实现了顺畅、成功的分割, 也对双路语 义分割网络运用于无人驾驶场景的可行性提供了 有力说明. 


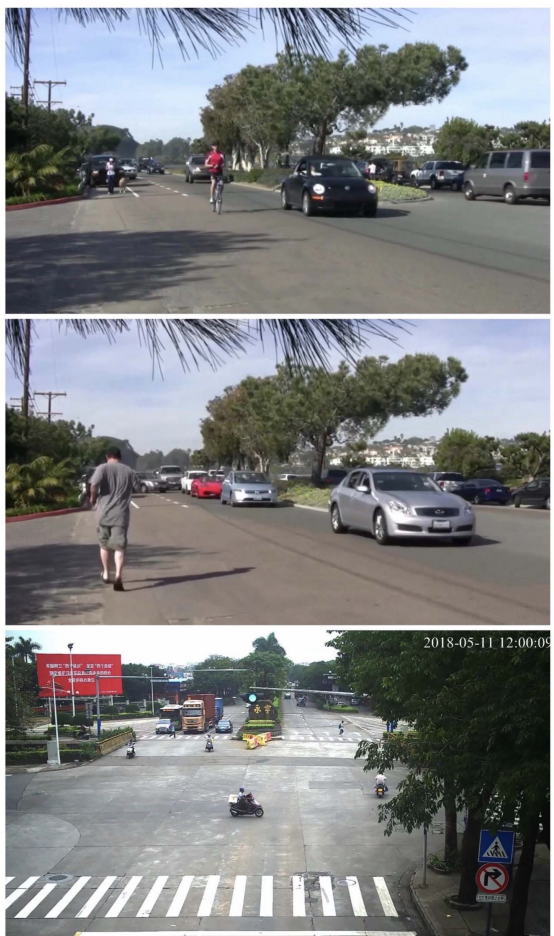

a. 原视频

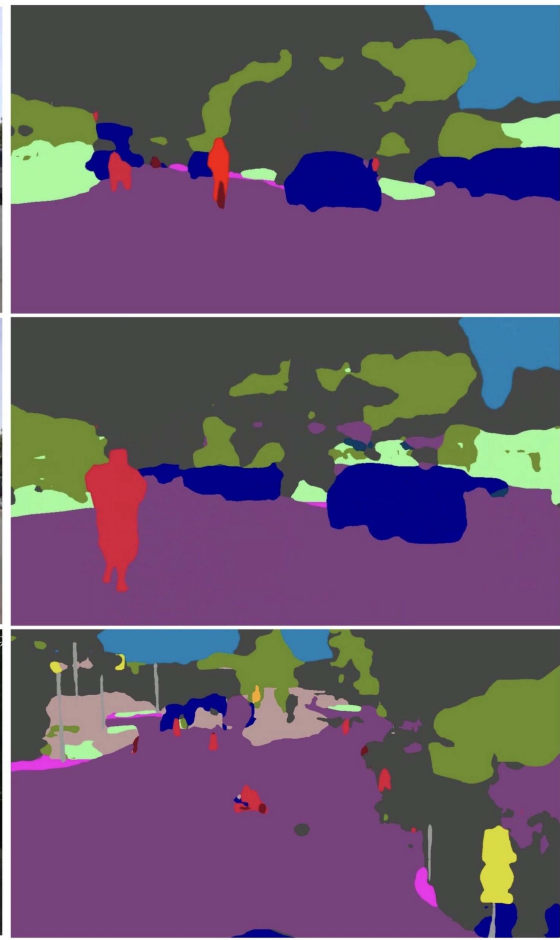

b. 双路语义分割网络的视频语义分割

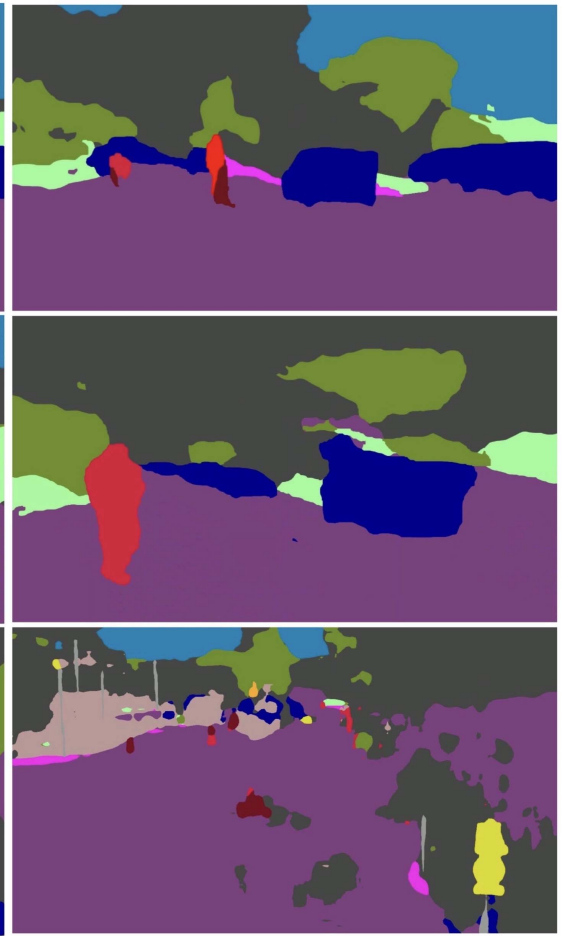

c. BiSeNet 的视频语义分割

图 8 部分视频语义分割关键帧

\section{5 结 语}

本文提出了双路语义分割网络, 能应用于无 人驾驶中的场景实时语义分割任务. 实验结果表 明, 它在 Cityscapes 数据集上获取了非常有竞争力 的效果, 超越了大多数现有网络. 针对道路语义分 割进行了要求和影响因素的分析, 提出在网络中 使用同一基础网络的不同分支分别获取空间信息 和上下文语义信息, 继而提出 ARM 用于优化上下 文语义信息分支的输出、提出 FFM 用于融合 2 分 支的输出, 最后, 选择主损失函数和双辅助损失函 数结合的方式进行网络优化. 在实验中, 采用针对 不同模块的有效性验证和网络整体性能分析的方 式, 充分验证了其有效性.

在结果可视化中发现, 存在的误分割中, 有部 分依照常识即可判断的误分割是可以避免的. 如 在一辆汽车的中间出现道路类别的识别、在路灯顶 部出现行人类别的识别等情况, 可以在后续工作 中加人先验知识训练模型, 从而减少误分割, 提高 整体精度.

\section{参考文献(References):}

[1] Huang Meiyi. A brief introduction on autonomous driving
technology[J]. Science \& Technology Information, 2017, 15(27): 1-2(in Chinese)

(黄美宜. 浅谈汽车无人驾驶技术 [J]. 科技资讯, 2017， 15(27): 1-2)

[2] Otsu N. A threshold selection method from gray-level histograms[J]. IEEE Transactions on Systems, Man, and Cybernetics, 1979, 9(1): 62-66

[3] Ge Milei. A technology of image segmentation based on cloud theory[D]. Harbin: Harbin Engineering University, 2010(in Chinese)

(葛秘蕾. 基于云理论的图像分割技术研究[D]. 哈尔滨: 哈 尔滨工程大学, 2010)

[4] Boykov Y Y, Jolly M P. Interactive graph cuts for optimal boundary \& region segmentation of objects in N-D images[C] //Proceedings of the 8th IEEE International Conference on Computer Vision. Los Alamitos: IEEE Computer Society Press, 2001: 105-112

[5] Rother C, Kolmogorov V, Blake A. "GrabCut": interactive foreground extraction using iterated graph cuts[J]. ACM Transactions on Graphics, 2004, 23(3): 309-314

[6] Long J, Shelhamer E, Darrell T. Fully convolutional networks for semantic segmentation[C] //Proceedings of the IEEE Conference on Computer Vision and Pattern Recognition. Los Alamitos: IEEE Computer Society Press, 2015: 3431-3440

[7] Krizhevsky A, Sutskever I, Hinton G E. ImageNet classification with deep convolutional neural networks[C] //Proceedings of Advances in Neural Information Processing Systems. Cambridge: MIT Press, 2012: 1097-1105

[8] Badrinarayanan V, Kendall A, Cipolla R. SegNet: a deep convolutional encoder-decoder architecture for image segmentation[J]. IEEE Transactions on Pattern Analysis and Machine 
Intelligence, 2017, 39(12): 2481-2495

[9] Chen L C, Papandreou G, Kokkinos I, et al. DeepLab: semantic image segmentation with deep convolutional nets, atrous convolution, and fully connected CRFs[J]. IEEE Transactions on Pattern Analysis and Machine Intelligence, 2017, 40(4): 834848

[10] Lin G S, Milan A, Shen C H, et al. RefineNet: multi-path refinement networks for high-resolution semantic segmentation[C] //Proceedings of the IEEE Conference on Computer Vision and Pattern Recognition. Los Alamitos: IEEE Computer Society Press, 2017: 1925-1934

[11] Zhao H H, Shi J P, Qi X J, et al. Pyramid scene parsing network[C] //Proceedings of the IEEE Conference on Computer Vision and Pattern Recognition. Los Alamitos: IEEE Computer Society Press, 2017: 2881-2890

[12] Paszke A, Chaurasia A, Kim S, et al. ENet: a deep neural network architecture for real-time semantic segmentation[OL]. [2020-08-27]. http://arxiv.org/pdf/1606.02147.pdf

[13] Zhao H S, Qi X J, Shen X Y, et al. ICNet for real-time semantic segmentation on high-resolution images[C] //Proceedings of the European Conference on Computer Vision. Heidelberg: Springer, 2018: 405-420

[14] Zhang X Y, Zhou X Y, Lin M X, et al. ShuffleNet: an extremely efficient convolutional neural network for mobile devices[C] //Proceedings of the IEEE Conference on Computer Vision and Pattern Recognition. Los Alamitos: IEEE Computer Society Press, 2018: 6848-6856

[15] Cowrdts M, Omran M, Ramos S, et al. The Cityscapes dataset for semantic urban scene understanding $[\mathrm{C}] / /$ Proceedings of the IEEE Conference on Computer Vision and Pattern Recognition. Los Alamitos: IEEE Computer Society Press, 2016: 3213-3223

[16] He K M, Zhang X Y, Ren S Q, et al. Deep residual learning for image recognition[C] //Proceedings of the IEEE Conference on Computer Vision and Pattern Recognition. Los Alamitos: IEEE Computer Society Press, 2016: 770-778

[17] He K M, Zhang X Y, Ren S Q, et al. Spatial pyramid pooling in deep convolutional networks for visual recognition[J]. IEEE
Transactions on Pattern Analysis and Machine Intelligence, 2015, 37(9): 1904-1916

[18] Hu J, Shen L, Sun G. Squeeze-and-excitation networks[C] //Proceedings of the IEEE Conference on Computer Vision and Pattern Recognition. Los Alamitos: IEEE Computer Society Press, 2018: 7132-7141

[19] Shrivastava A, Gupta A, Girshick R. Training region-based object detectors with online hard example mining[C] //Proceedings of the IEEE Conference on Computer Vision and Pattern Recognition. Los Alamitos: IEEE Computer Society Press, 2016: 761-769

[20] Yu F, Koltun V. Multi-scale context aggregation by dilated convolions[OL]. [2020-08-27]. http://arxiv.org/pdf/1511.07122.pdf

[21] Ghiasi G, Fowlkes C C. Laplacian pyramid reconstruction and refinement for semantic segmentation[C] //Proceedings of European Conference on Computer Vision. Heidelberg: Springer, 2016: 519-534

[22] Li H C, Xiong P F, Fan H Q, et al. DFANet: deep feature aggregation for real-time semantic segmentation[C]//Proceedings of the IEEE Conference on Computer Vision and Pattern Recognition. Los Alamitos: IEEE Computer Society Press, 2019: 9522-9531

[23] Wang P Q, Chen P F, Yuan Y, et al. Understanding convolution for semantic segmentation[C] //Proceedings of the IEEE Winter Conference on Applications of Computer Vision. Los Alamitos: IEEE Computer Society Press, 2018: 1451-1460

[24] Yu C Q, Wang J B, Peng C, et al. BiSeNet: bilateral segmentation network for real-time semantic segmentation[C] //Proceedings of the European Conference on Computer Vision. Heidelberg: Springer, 2018: 325-341

[25] Wu Z F, Shen C H, van den Hengel A. Real-time semantic image segmentation via spatial sparsity[OL]. [2020-08-27]. http://arxiv.org/pdf/1712.00213.pdf

[26] Treml M, Arjona-Medina J, Unterthiner T, et al. Speeding up semantic segmentation for autonomous driving $[\mathrm{C}]$ //Proceedings of the 29th Conference on Neural Information Processing Systems. Cambridge: MIT Press, 2016: 2-7 